\section{Primary Sjögren Syndrome in a Child with a Neuromyelitis Optica Spectrum Disorder}

To the Editor:

Neuromyelitis optica spectrum disorders (NMOSD) are a group of rare autoimmune diseases typically affecting women in their 40s and 50s, as does Sjögren syndrome (SS), another autoimmune disease. We describe a 6-year-old girl who presented with clinical and laboratory findings of both an NMOSD and SS.

A 6-year-old previously healthy Korean girl presented with a 1-day history of fever, headache, progressive right-sided weakness, and altered mental status. Cerebrospinal fluid (CSF) analysis revealed an elevated protein without pleocytosis or oligoclonal bands. Magnetic resonance imaging (MRI) of the spinal cord showed contiguous T2 hyperintensity, and MRI of the brain showed extensive white matter lesions bilaterally (Figure 1), suspicious for demyelinating disease. She was treated with high-dose methylprednisolone, resulting in marked improvement, and continued treatment with tapering doses of prednisone, but flared when the prednisone was discontinued.

CSF was positive by ELISA for neuromyelitis optica autoantibody (NMO)-immunoglobulin $\mathrm{G}(\operatorname{IgG})$, as was the serum when subsequently tested. Blood was also positive for antinuclear antibody (ANA) at 1:160 (speckled) and anti-SSA (Ro) antibody. Anti-SSB (La), anti-dsDNA, anti-Sm, and anti-RNP antibodies, rheumatoid factor, and complement factor 3 (C3) and $\mathrm{C} 4$ were negative or normal. The patient also started receiving azathioprine (AZA) as a steroid-sparing agent.

The patient had no symptoms of SS, such as mouth or eye dryness, joint pain, or parotitis. An ophthalmological examination was normal. However, a minor salivary gland biopsy revealed $>1$ lymphocytic aggregate per $4 \mathrm{~mm}^{2}$, consistent with a diagnosis of SS. Based on studies showing its efficacy in treating both SS and NMOSD ${ }^{1,2}$, rituximab (RTX) was started, after which the prednisone and AZA were discontinued. Repeat MRI demonstrated improvement and no new lesions. Three years after presentation, continued treatment with RTX every 6 months and taking no other medica- tions, the patient displayed only subtle right-sided weakness and mild abducens and facial nerve weakness on examination.

NMOSD are rare in children and account for only $3.2 \%-8.5 \%$ of central nervous system (CNS) demyelinating disease under the age of $16^{2}$. Discovery of a serum IgG autoantibody against the astrocytic water channel aquaporin-4 (AQP4) in this disorder has improved understanding of the range of NMOSD. While clinical NMOSD are present in $92 \%$ of adults with the anti-NMO antibody (NMO-IgG), essentially all children with the antibody showed recurrent disease along the continuum of NMOSD, including recurrent optic neuritis and recurrent longitudinally extensive transverse myelitis ${ }^{3}$. As a result, the NMOSD have been redefined clinically and NMO-IgG has been increasingly used to establish the diagnosis of an NMOSD in children ${ }^{4,5}$. With acute myelitis, positive NMO-IgG, and exclusion of alternative diagnoses, our patient met the recently proposed international criteria for $\mathrm{NMOSD}^{5}$

Primary SS in children, though rare, has been reported ${ }^{6,7}$. SS is characterized by plasma cell and lymphocyte infiltration of the exocrine glands, classically resulting in clinical xerostomia and xerophthalmia. In children, however, SS often presents insidiously, most commonly with recurrent parotid swelling, but also with a broad spectrum of symptoms outside of the typical sicca syndrome seen in adults ${ }^{6,7}$. CNS involvement is well documented in both pediatric and adult patients with $\mathrm{SS}^{8}$. Patients with SS usually have anti-SSA and/or anti-SSB antibodies, but the presence of these antibodies may not be of diagnostic significance and may be present in completely asymptomatic individuals. Because of the lack of typical symptoms in pediatric SS, diagnosis is often difficult.

NMOSD have a high prevalence of coexistent autoimmune disorders and markers. Over $40 \%$ of adult patients with NMOSD have comorbid autoimmune diseases and over $75 \%$ have autoantibodies aside from NMOIgG: as many as $64 \%$ also have a positive ANA and $15 \%-38 \%$ have a positive $\mathrm{SSA}^{4}$.

Interestingly, while there are multiple reports of coexisting SS and NMOSD in adults ${ }^{9}$, to our knowledge, this has not been definitively reported in pediatrics. Several patients with NMOSD have been reported to have
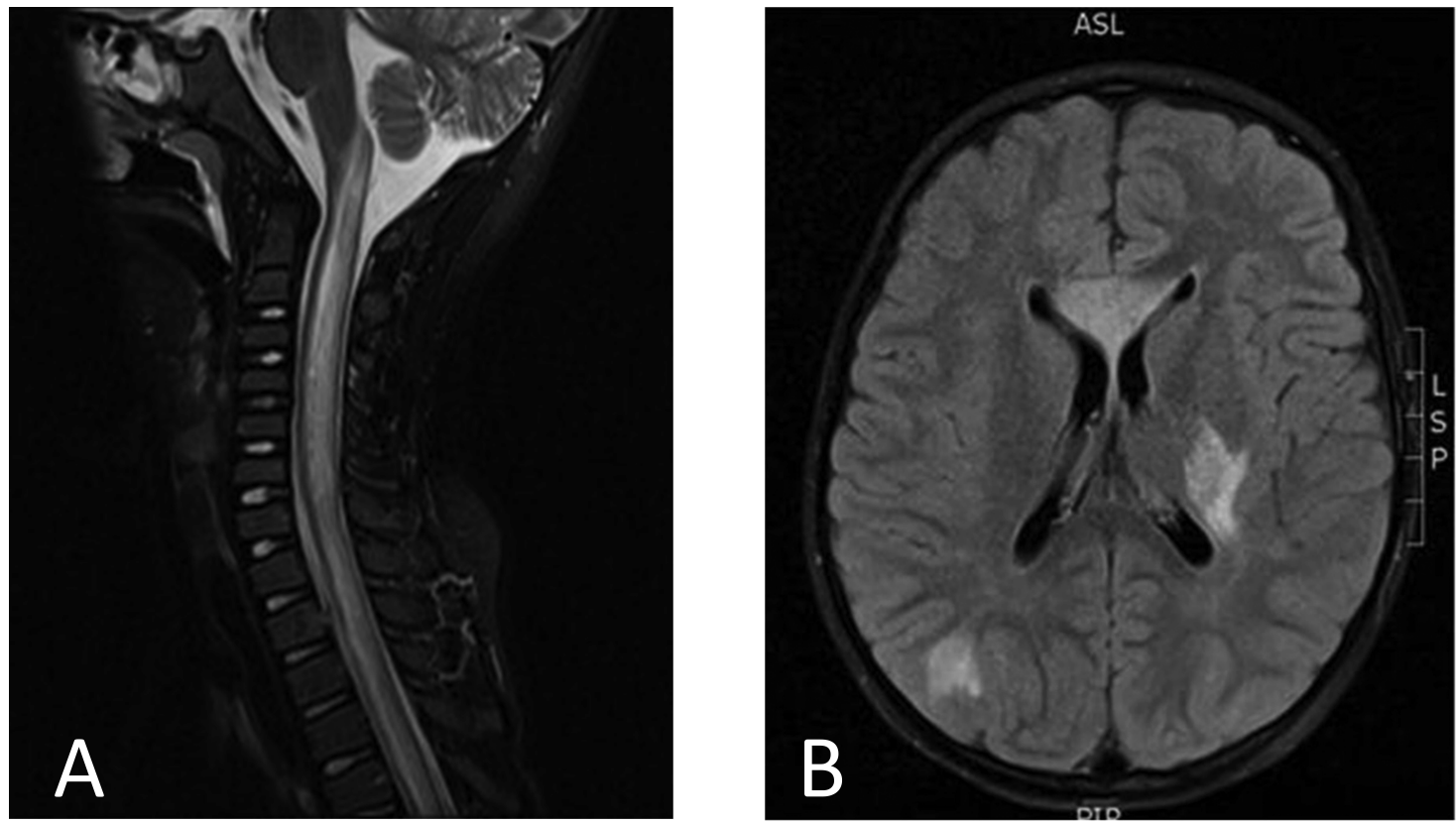

Figure 1. A. MRI of the cervical and thoracic spine (T2 FLAIR with contrast shown) obtained at the onset of symptoms, prior to treatment. There is a contiguous hyperintense, centrally located spinal cord lesion. B. MRI of the head (T2 FLAIR with contrast shown) obtained at the onset of symptoms, prior to treatment. There are multiple lesions of the supratentorial brain (especially involving the white matter). MRI: magnetic resonance imaging; FLAIR: fluid attenuated inversion recovery.

Personal non-commercial use only. The Journal of Rheumatology Copyright @ 2016 . All rights reserved. 
positive anti-SSA. While these patients may also have had SS, they either did not otherwise meet diagnostic criteria for SS (because of the lack of salivary gland biopsies) $)^{10}$ or specific details concerning SS diagnosis were not published ${ }^{4}$. Clinical and imaging findings possibly consistent with an NMOSD along with definitive SS have also been reported, but the diagnosis of an NMOSD was not confirmed because no NMO-IgG results were reported $^{8,11}$.

An intrinsic link between SS and NMOSD has been suggested ${ }^{12}$. The human salivary glands possess aquaporin-3 and aquaporin-5 channels ${ }^{13}$; AQP4 channels have been found in rodent salivary glands ${ }^{14}$. Autoimmunity in the salivary gland, in the context of SS, may result in an immune response against AQP $4^{12}$. Previous studies have suggested that patients with SS with neurologic deficits should have an examination for NMOSD ${ }^{15}$. Conversely, our case suggests that patients presenting with NMOSD should be evaluated for the possibility of SS, and in addition highlights the difficulties inherent in detecting and diagnosing SS in children.

JEFFREY M. KORNITZER, MD, Department of Neurology, Rutgers-New Jersey Medical School, Newark, New Jersey, USA; YUKIKO KIMURA, MD, Section Chief, Section of Pediatric Rheumatology, Joseph M. Sanzari Children's Hospital, Hackensack University Medical Center, Hackensack, New Jersey, USA; GINGER L. JANOW, MD, MPH, Section of Pediatric Rheumatology, Joseph M. Sanzari Children's Hospital, Hackensack University Medical Center, Hackensack, New Jersey, USA. Address correspondence to Dr. G. Janow, Section of Pediatric Rheumatology, Joseph M. Sanzari Children's Hospital, Hackensack University Medical Center, 30 Prospect Ave., WFAN Bldg. 3rd Floor, Hackensack, New Jersey 07601, USA.E-mail: GJanow@HackensackUMC.org

\section{REFERENCES}

1. Meijer JM, Meiners PM, Vissink A, Spijkervet FK, Abdulahad W, Kamminga N, et al. Effectiveness of rituximab treatment in primary Sjogren's syndrome: a randomized, double-blind, placebo-controlled trial. Arthritis Rheum 2010;62:960-8.

2. Longoni G, Banwell B, Filippi M, Yeh EA. Rituximab as a first-line preventive treatment in pediatric NMOSDs: preliminary results in 5 children. Neurol Neuroimmunol Neuroinflamm 2014;1:e46.

3. Banwell B, Tenembaum S, Lennon VA, Ursell E, Kennedy J, Bar-Or A, et al. Neuromyelitis optica-IgG in childhood inflammatory demyelinating CNS disorders. Neurology 2008;70:344-52.

4. McKeon A, Lennon VA, Lotze T, Tenenbaum S, Ness JM, Rensel $\mathrm{M}$, et al. CNS aquaporin-4 autoimmunity in children. Neurology 2008;71:93-100.
5. Wingerchuk DM, Banwell B, Bennett JL, Cabre P, Carroll W, Chitnis T, et al; International Panel for NMO Diagnosis. International consensus diagnostic criteria for neuromyelitis optica spectrum disorders. Neurology 2015;85:177-89.

6. Cimaz R, Casadei A, Rose C, Bartunkova J, Sediva A, Falcini F, et al. Primary Sjogren syndrome in the paediatric age: a multicentre survey. Eur J Pediatr 2003;162:661-5.

7. Movva S, Carsons S. Review of pediatric Sjogren's syndrome. Pediatr Allergy Immunol Pulmonol 2014;27:111-1146.

8. Arabshahi B, Pollock AN, Sherry DD, Albert DA, Kreiger PA, Pessler F. Devic disease in a child with primary Sjogren syndrome. J Child Neurol 2006;21:285-6.

9. Carvalho DC, Tironi TS, Freitas DS, Kleinpaul R, Talim NC, Lana-Peixoto MA. Sjögren syndrome and neuromyelitis optica spectrum disorder co-exist in a common autoimmune milieu. Arq Neuropsiquiatr 2014;72:619-24

10. Lotze TE, Northrop JL, Hutton GJ, Ross B, Schiffman JS, Hunter JV. Spectrum of pediatric neuromyelitis optica. Pediatrics 2008;122:e1039-47.

11. Yokogawa N, Lieberman SM, Alawi F, Bout-Tabaku S, Guttenberg M, Sherry DD, et al. Comparison of labial minor salivary gland biopsies from childhood Sjogren syndrome and age-matched controls. J Rheumatol 2014;41:1178-82.

12. Graber DJ, Levy M, Kerr D, Wade WF. Neuromyelitis optica pathogenesis and aquaporin 4. J Neuroinflammation 2008;5:22.

13. Gresz V, Kwon TH, Hurley PT, Varga G, Zelles T, Nielsen S, et al. Identification and localization of aquaporin water channels in human salivary glands. Am J Physiol Gastrointest Liver Physiol 2001;281:G247-54.

14. Mobasheri A, Marples D, Young IS, Floyd RV, Moskaluk CA, Frigeri A. Distribution of the AQP4 water channel in normal human tissues: protein and tissue microarrays reveal expression in several new anatomical locations, including the prostate gland and seminal vesicles. Channels 2007;1:29-38.

15. Wingerchuk DM, Weinshenker BG. The emerging relationship between neuromyelitis optica and systemic rheumatologic autoimmune disease. Mult Scler 2012;18:5-10.

J Rheumatol 2016;43:6; doi:10.3899/jrheum.151207

Personal non-commercial use only. The Journal of Rheumatology Copyright (C) 2016. All rights reserved. 